\title{
A simple volume and energy- dependent form for the Grüneisen parameter of silicate liquids
}

\author{
PAUl D. ASIMOW ${ }^{1}$ AND YACONG (BROOKE) ZHOU ${ }^{1}$
}

${ }^{1}$ Division of Geological and Planetary Sciences, California Institute of Technology, Pasadena CA 91125 USA, asimow@gps.caltech.edu

Understanding melting in the deep interior of terrestrial planet mantles, e.g. in an evolving magma ocean or perhaps at the modern core mantle boundary, requires an accurate and easy-to-use expression for the thermal Equation of State (EoS) of multicomponent silicate liquids. The functional form of such an EoS is still a matter of research. Data density and simulation quality are reaching the point that several assumptions made in EOS formulations can now be tested or generalized. In particular, the thermal pressure is often described by a Grüneisen parameter $(\gamma)$, which is commonly assumed to be a one-parameter function of volume $(V)$. This is called the Mie-Grüneisen approximation and is wellmotivated for solid materials. For liquids it is questionable. Two previous molecular dynamics (MD) studies of anorthite liquid $[1,2]$ have shown hints of systematic deviation from Mie-Grüneisen, without detailed investigation. Our new campaign of combined empirical (1108 atoms) and ab initio (277 atoms) MD simulations of the Diopside-Anorthite (DiAn) eutectic liquid composition have shown clear and systematic dependence of $\gamma$ on internal energy $(E)$ at constant $V$ as well as the exotic $V$-dependence previously emphasized. We abstracted a simple functional form with only one extra parameter from these simulations, which also show systematic variation in liquid isochoric heat capacity $\left(C_{V}\right)$ and suggest a functional form for $C_{V}$. Together, these yield a new and more predictive thermal EoS in $P-V-E-T$ space that has few enough parameters to be fit to very sparse experimental data. The resulting experimental fit is only weakly dependent on inaccuracies and uncertainties in the simulations.

By shock compression, it is possible to directly measure density, temperature, and sound speed in silicate liquids at any mantle pressure $(P)$. The new functonal form is able to fit the combined sound-speed and shock velocity data set for all starting phases and temperatures in the DiAn system. The new model makes updated predictions of the shape of magma ocean liquid isentropes. Generalization to other components and the ideal mixing model allow a new look at the location of initial crystallization and the buoyancy of crystals.

[1] M. S. Ghiorso et al. (2009) GCA 73:6937-6951.

[2] N. de Koker (2010) GCA 74:5657-5671. 